\title{
SURVEY FOR TRANSITING EXTRASOLAR PLANETS IN STELLAR SYSTEMS. IV. VARIABLES IN THE FIELD OF NGC 1245
}

\author{
Joshua Pepper and Christopher J. Burke \\ Department of Astronomy, Ohio State University, Columbus, OH 43210; pepper@astronomy.ohio-state.edu, cjburke@astronomy.ohio-state.edu \\ Received 2006 April 4; accepted 2006 May 9
}

\begin{abstract}
The Survey for Transiting Extrasolar Planets in Stellar Systems (STEPSS) project is a search for planetary transits in open clusters. In this paper we analyze the STEPSS observations of the open cluster NGC 1245 to determine the variable-star content of the cluster. Out of 6787 stars observed with $V<22$, of which $\sim 870$ are cluster members, we find 14 stars with clear intrinsic variability that are potential cluster members and 29 clear variables that are not cluster members. None of these variables have been previously identified. We present light curves, finder charts, and stellar/ photometric data on these variable objects. Several of the interacting binaries have estimated distances consistent with the cluster distance determined from isochrone fits to the color-magnitude diagram. Four stars at the main-sequence turnoff of the cluster have light curves consistent with $\gamma$ Doradus variability. If these $\gamma$ Doradus candidates are confirmed, they represent the oldest and coolest members of this class of variable discovered to date.
\end{abstract}

Key words: binaries: general — stars: variables: other - surveys — techniques: photometric

Online material: extended figure sets

\section{INTRODUCTION}

The dramatic increase in the number of surveys for planetary transits over the last 5 years has resulted in a wealth of highprecision stellar photometry (Horne 2003). Although primarily conducted to detect planetary transits, such surveys also produce data sets that allow for intensive investigation of stellar variability. Especially useful are surveys of clusters, which can be used to characterize the variability content of the cluster. Detections of eclipsing binaries can provide a check on the cluster's distance, as well as helping to characterize the mass-radius and massluminosity relationships.

Many surveys of clusters have already published data sets of high-precision photometry, finding low-amplitude variables, eclipsing binaries, and other pulsating stars. The Planets in Stellar Clusters Extensive Search project has observed clusters NGC 2158 and NGC 6791 (Mochejska et al. 2002, 2004, 2005). The Extrasolar Planet Occultation Research/Open Clusters project has observed clusters NGC 2660 and NGC 6208 (von Braun et al. 2005). Other projects have observed or are observing NGC 7789 , 6819, and 6940 (Street et al. 2003; Bramich et al. 2005), NGC 6633 (Hidas et al. 2005), and NGC 6705 (Hargis et al. 2004).

The Survey for Transiting Extrasolar Planets in Stellar Systems (STEPSS; Burke et al. 2002) concentrates on searching open clusters for planetary transits. Searching for transits in clusters is advantageous, since cluster members have similar metallicities and ages and are therefore good laboratories for determining the distribution of planets without varying those measurable properties. Here we present an analysis of the observations of NGC 1245 to catalog the stellar variables in the cluster.

\section{CLUSTER PARAMETERS AND OBSERVATIONS}

See Burke et al. (2004) for a full discussion of the parameters of the cluster. NGC 1245 is a rich open cluster with an age of $1.04 \pm 0.09$ Gyr. It has a metallicity slightly less than solar, $[\mathrm{Fe} / \mathrm{H}]=-0.05 \pm 0.08$. The cluster is at a distance of $2.8 \pm$ $0.2 \mathrm{kpc}$, with a distance modulus of $(m-M)_{0}=12.27 \pm 0.12$. The core radius is $r_{c}=3$ ' $^{\prime} 10 \pm 0.52(2.57 \pm 0.43 \mathrm{pc})$. The total cluster mass is $M=2700 \pm 600 M_{\odot}$. All errors given above are systematic and are larger than the statistical errors.
The STEPSS project observed NGC 1245 over the course of 19 nights in 2001 October and November. The observations were obtained with the MDM 8K mosaic imager on the MDM $2.4 \mathrm{~m}$ Hiltner telescope, yielding a $26^{\prime} \times 26^{\prime}$ field of view with 0 "' 36 resolution per pixel. In total, the data set consists of 936 I-band images, with typical exposure times of $300 \mathrm{~s}$. For the color-magnitude diagram (CMD), several supplementary $B$ - and $V$-band images were also obtained. None of the nights were photometric, and so a second series of observations was taken in 2002 February to calibrate the cluster photometry.

\section{LIGHT CURVES AND STELLAR PROPERTIES}

We follow the procedure outlined in Burke et al. (2005) to generate light curves, which develops a new method for differential photometry given the demands of detecting transiting extrasolar planets $(<0.01 \mathrm{mag}$ precision $)$. The main features of the method include allowing each star to have a unique comparison ensemble, minimization of the light-curve standard deviation as a figure of merit, and full automation. Applying this method yields light curves that have precision to less than $1 \%$ for $V<18$.

Of the 6787 stars identified in the survey's field of view, we estimate that $\sim 870$ belong to the cluster. This estimate comes from scaling and subtracting star counts from a control field on the outskirts of our field of view. Figure 1 shows the $(V, B-V)$ CMD of the cluster field along with the best-fit isochrone from Burke et al. (2004), derived from the theoretical isochrones of Yi et al. (2001). The CMD shown here uses the data from which the light curves were derived, while a different data set that avoided saturation on the brighter stars was used to determine cluster properties such as the turnoff point.

Given the cluster age, metallicity, distance, and reddening, the best-fit isochrone transforms an observed apparent magnitude into the stellar properties, assuming the star is a cluster member. Using the stellar mass as the independent variable, we determine the stellar mass that minimizes the $\chi^{2}$ distance between the apparent magnitude and the best-fit isochrone magnitudes in the $B V I$ passbands.

A caveat for assigning stellar properties based on the CMD is the assumption that the detected stellar light arises from a single 


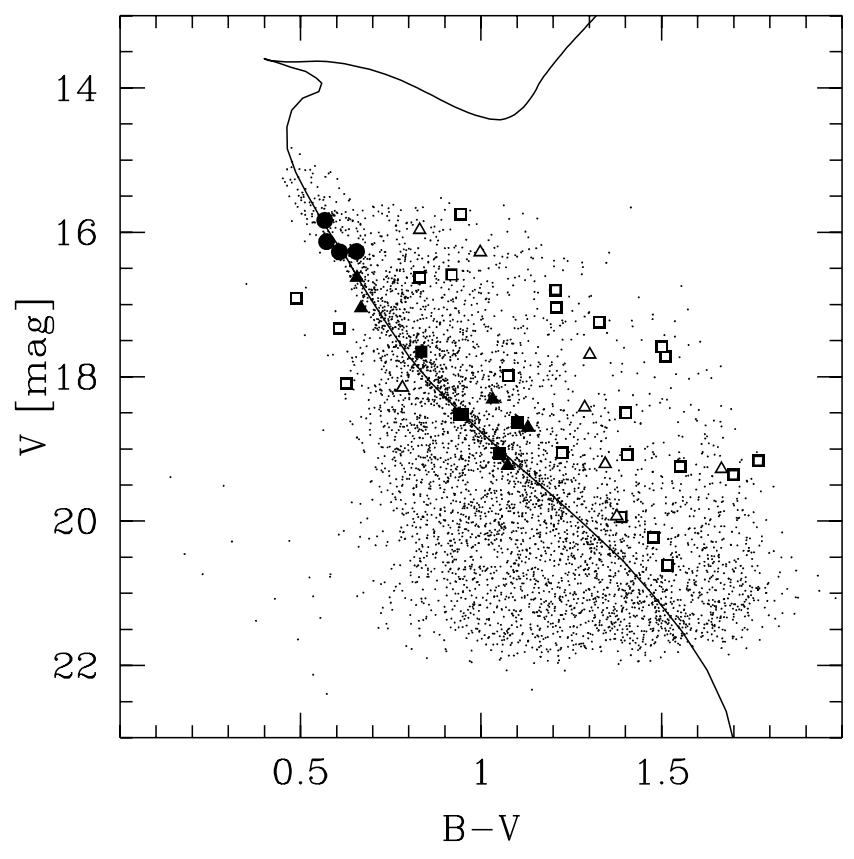

FIG. 1.- $(V, B-V) \mathrm{CMD}$ for NGC 1245 . The best-fit isochrone to the cluster main sequence is shown with a solid line. Filled symbols represent cluster variables, while open symbols represent field variables. The triangles show binary variables (see $\S 5.1$ ), the circles show $\gamma$ Doradus candidates (see $\S 5.2$ ), and the squares represent unclassified variables.

main-sequence cluster member. The stellar properties contain significant systematic errors if the object does not belong to the cluster or if a portion of the stellar light arises from an unresolved stellar companion. The physical properties for the variable sources detected in this study should be regarded with extra caution, considering that most sources of variability result from the binary nature of an object (Sterken \& Jaschek 1996) and thus have a large probability for significant stellar companion contamination.

\section{VARIABLE-STAR SELECTION}

One of the great challenges in determining variability with high-precision photometry is the difficulty of distinguishing intrinsic variability from noise and systematic errors. Common classes of variable stars demonstrate both large amplitude variations and periodic behavior, making them both easily detected and easily characterized. However, with the advent of surveys that observe thousands of stars with millimagnitude photometry, robustly detecting variations at low noise levels requires a variety of statistical selection criteria. Some surveys, such as the All Sky Automated Survey (Eyer \& Blake 2005) and the Optical Gravitational Lensing Experiment (Mizerski \& Bejger 2002), have developed automated classification pipelines. Many surveys, however, use a few simple statistical cuts to identify variable stars independent of the source or type of variability.

Our variability selection routine has three stages. First, we eliminate outlying measurements and require a minimum number of remaining measurements. Second, we compute three different statistics that describe the behavior of the light curve: the rms, the Stetson $J$-statistic (Stetson 1996), and the analysis of variance (AoV) periodicity statistic (Schwarzenberg-Czerny 1996). We apply cuts on each of those values to select variable candidates. Third, we visually inspect the light curves and images of the variable candidates to eliminate false-positive candidates with variability due to blending effects from nearby objects or detector defects. In $\S 4.4$ we describe two tiers of statistical cuts. The first, more stringent cut does not contain any false positives. A second, less stringent cut with higher sensitivity contains lower amplitude variables but also contains $<7 \%$ false positives, which are later eliminated through visual inspection.

\subsection{Eliminating Outlying Points}

The first stage of the analysis removes statistically outlying photometric measurements. Outliers are identified in the following manner: For each star we calculate the reduced $\chi^{2}$ light-curve variability,

$$
\chi^{2}=\frac{1}{n-1} \sum_{k=1}^{n} \frac{\left(m_{k}-\mu\right)^{2}}{\sigma_{k}^{2}}
$$

where the sum is over $n$ observations, with magnitude $m_{k}$, with er$\operatorname{ror} \sigma_{k}$, and $\mu$ is the weighted average magnitude of the light curve,

$$
\mu=\frac{\sum_{k=1}^{n} m_{k} \sigma_{k}^{-2}}{\sum_{k=1}^{n} \sigma_{k}^{-2}}
$$

The $\chi^{2}$ statistic measures the degree to which a constant lightcurve model approximates the light-curve data within the context of the measurement errors. Light curves with intrinsic variability result in high $\chi^{2}$ values, denoting that a constant light-curve model poorly represents the data. Before eliminating outlying measurements we scale the errors by setting $\sigma_{k}^{\prime 2}=\sigma_{k}^{2} \chi^{2}$, thus enforcing $\chi^{2}=1$. The error scaling allows the Gaussian noise, systematic error, or intrinsic variability (whichever noise source dominates the scatter in the light curve) to set the scale for judging whether a measurement is a statistical outlier. This procedure can eliminate intrinsic variability that affects a small number of the measurements. This hampers our ability to detect largeamplitude eclipsing binaries when only a single eclipse occurred during the observations. However, given our quantitative selection criteria and documented analysis techniques, these biases can easily be accounted for with a more detailed inspection of the data.

For each observation, we compute the deviation, $\delta_{k}=\mid m_{k}-$ $\mu \mid / \sigma_{k}^{\prime}$. All photometric measurements with $\delta_{k}>3$ are eliminated. We perform three iterations of the above procedure for each light curve. The light curves with $<750$ measurements remaining typically result from false stellar detections near the cores of bright isolated stars and are discarded. We also eliminate light curves with rms $<0.3$ mag, since no light curves with higher scatter show any kind of coherent intrinsic variability.

\subsection{The Stetson J-Statistic}

The Stetson $J$-statistic (Stetson 1996) provides our primary measure of the coherent intrinsic light-curve variability. The statistic measures variability by weighting photometric variations that are correlated in time. A light curve with a steady change in brightness over a period of time results in a higher $J$-value than a curve with Gaussian noise, even though both curves may have equal values for $\chi^{2}$. The statistic is defined by

$$
J=\frac{\sum_{k=1}^{n} w_{k} \operatorname{sgn}\left(P_{k}\right) \sqrt{\left|P_{k}\right|}}{\sum_{k=1}^{n} w_{k}},
$$

where the observer defines $n$ pairs of observations with weights $w_{k}$. We define observations as a pair when they are separated by 

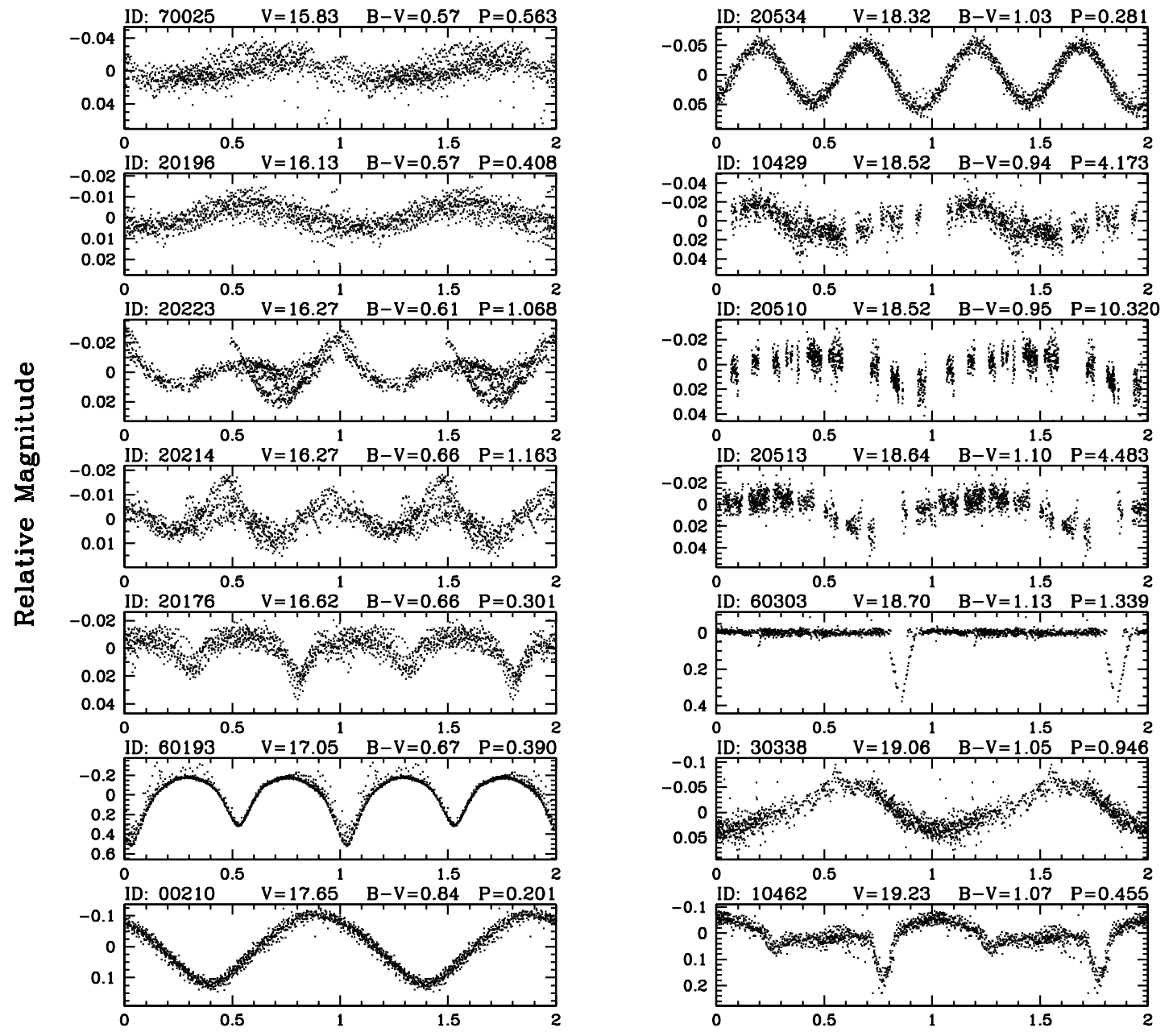

\section{Phase}

FIG. 2.-Phased light curves for variables that are potential cluster members, sorted by apparent magnitude.

less than 0.02 days; $P_{k}$ is the product of the normalized residuals of two observations $i$ and $j$, constituting the $k$ th pair, such that

$$
P_{k}= \begin{cases}\delta_{i(k)} \delta_{j(k)}, & i(k) \neq j(k), \\ \delta_{i(k)}^{2}-1, & i(k)=j(k),\end{cases}
$$

and $\delta$ is the relative error, defined as

$$
\delta=\sqrt{\frac{n}{n-1}} \frac{m-\mu}{\sigma},
$$

where $m$ is the apparent magnitude of the star, $\sigma$ is the error in the magnitude, and $\mu$ is the weighted mean magnitude of the star. Following the choices of Kaluzny et al. (1998), we set $w_{k}=1$ for pairs of observations $[i(k) \neq j(k)]$ and $w_{k}=0.25$ for single observations $[i(k)=j(k)]$, and we also multiply the final quantity $J$ by $\sum_{k=1}^{n} w_{k} / w_{\max }$, where $w_{\max }$ is the total weight the star would have if it were measured on all images. Although our typical cadence is 6.5 minutes, we allow pairs separated by up to $20 \%$ of the shortest periods we search for ( 0.1 days; see $\S 4.3$ ) to extend the usefulness of $J$ to find longer period variables.

Of the stars we observe, the Stetson statistic varies from $J \ll 1$ for nonvariable sources to $1<J<10$ for obvious variables. For objects with $J \approx 1$, it can be difficult to demarcate a specific cut in $J$ to specify variability. We describe our choices for cuts on $J$ in $\S 4.4$.

\subsection{Periodicity Analysis}

To select periodic variations, we apply the multiharmonic analysis of variances period-search algorithm (ANOVA) described by Schwarzenberg-Czerny (1996). Given a range of periods, the ANOVA algorithm produces a likelihood statistic, $0 \leq \mathrm{AoV} \leq 1$, that returns the quality of fit for orthonormal polynomials to the light-curve variability. Since the observations occurred over a duration of 19 days, we run the ANOVA algorithm over a period range of 0.1-14 days, which we believe to be the variability periods that we could reasonably detect.

One problem with applying the ANOVA algorithm to groundbased observations is the appearance of significant light-curve variability on the diurnal period and its aliases. The rise and set of the field of observation and the corresponding secular changing atmospheric path length and mechanical stresses on the telescope impart systematic errors in the light curve. Our light curves display trends in the light-curve variability as a function of seeing. We correct for these effects using the detrending algorithm described in the beginning of $\S 3$, but trends above the expected Gaussian noise remain. We find signs of aliasing primarily at periods of $\frac{1}{3}, \frac{1}{2}, \frac{2}{3}, 1,2,3$, and 4 days. Relying on the ANOVA statistic alone results in many detections of low-amplitude $(<0.01 \mathrm{mag})$ 

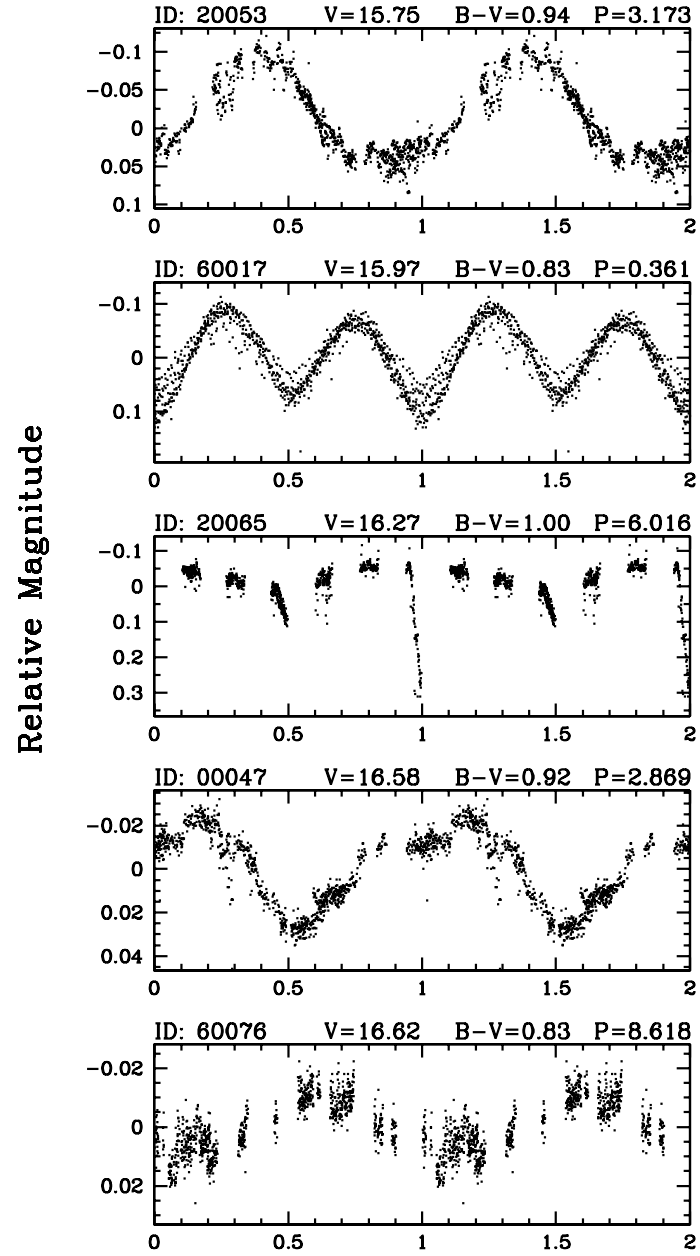
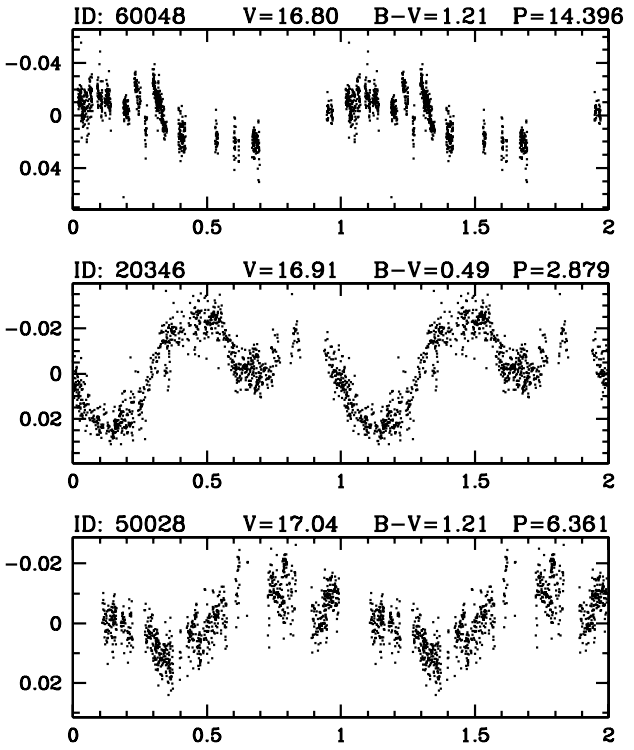

ID: $00036 \quad V=17.24 \quad B-V=1.33 \quad P=11.271$
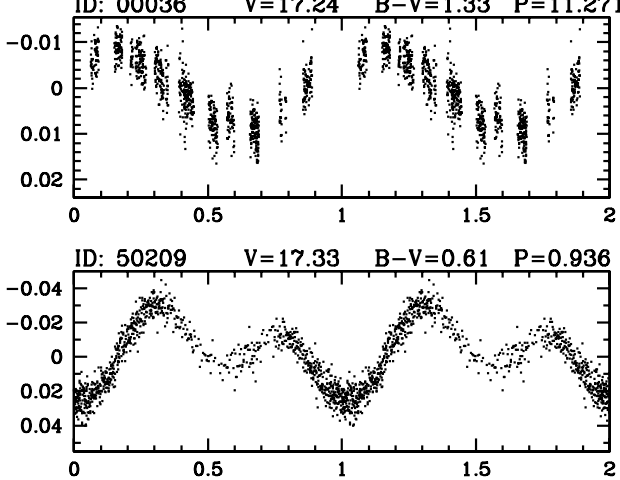

\section{Phase}

FIG. 3.-Phased light curves for field variables, sorted by apparent magnitude.

variables with periods at the diurnal period and its aliases. However, in combination with the Stetson $J$-statistic (see $\S 4.4$ ) these alias variables do not make it into the final variable object samples.

\subsection{Final Cuts and Visual Inspection}

There are several systematic effects that complicate the attempt to impose categorical cuts in $J$ and AoV that result in a sample that includes all probable intrinsic variables and zero false detections. Systematic errors in the light curve, such as blended objects, edge effects, detector defects, and detector saturation, can artificially inflate $J$ and AoV for nonvariable sources. Furthermore, such effects, along with poor photometry, can in some cases lead to lower $J$ and AoV values for true variables, compared to perfect photometry. We conclude that the most useful evaluation of the variability in our sample is a two-tier selection, in which we identify stars with robust variability and zero false detections, and then a second tier of candidate variables with a less stringent selection, resulting in a sample with some low contamination due to false detections.

Our first tier consists of stringent cuts on the $J$ and AoV statistical values. We find that cuts of $J>1.2$ and AoV $>0.65$ yield an ensemble of 23 stars that are all clearly intrinsic variables. The first tier has zero contamination due to false positives. However, it misses a number of other clear variables that vary with lower amplitudes. We then conduct a visual inspection of the images of the remaining sources and flag those that are near detector defects such as bad columns, diffraction spikes, and bleed trails. A second tier cut of $J>0.75$ and AoV $>0.55$ includes most stars in our sample that have coherently varying light curves and includes few false positives. This second cut contains 44 variable candidates, including all the first-tier objects. Visual inspection of the light curves and images of these stars enables us to eliminate three of the second-tier stars due to detection effects, yielding a contamination rate of $\sim 7 \%$. Based on a visual inspection of light curves, the contamination rate climbs rapidly for lower statistical cuts. On visual inspection of stars that do not make the cuts, we do find two more field stars that show coherent, periodic variability at low amplitudes. We include these two stars in our field star sample.

We show the light curves and photometric properties of the potential cluster members meeting both selection cuts in Figure 2. The plots for the field variables are in Figures 3-5. Additional detail on each cluster variable is shown in Figure Set 6 , and for the field variables in Figure Set 7. Figures 6 and 7 show the complete detail for objects 70025 and 20053, respectively, with the other objects online. We show the unphased light curve in panel $a$ for the full 19 days of the survey. In panel $b$ we show the phased light curve of the variable, with two periods plotted. In the top right corner is a finder chart showing the variable marked with white bars. The nearest detected object is marked 

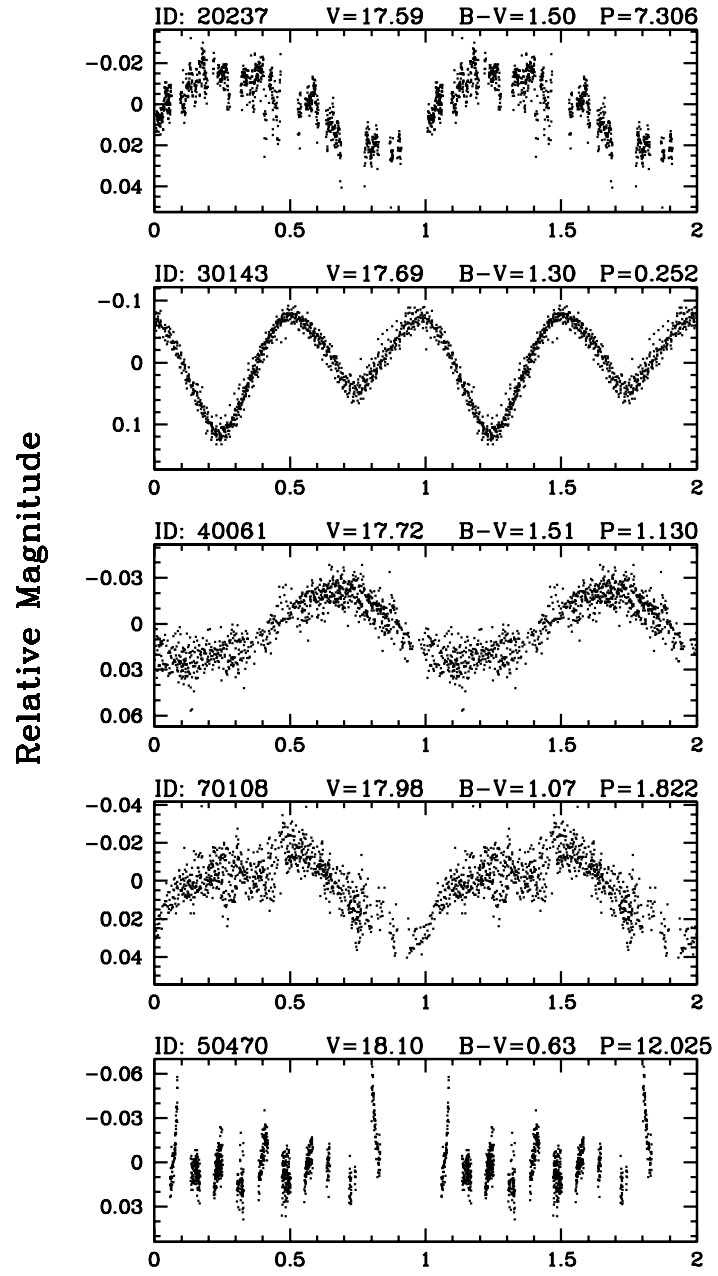
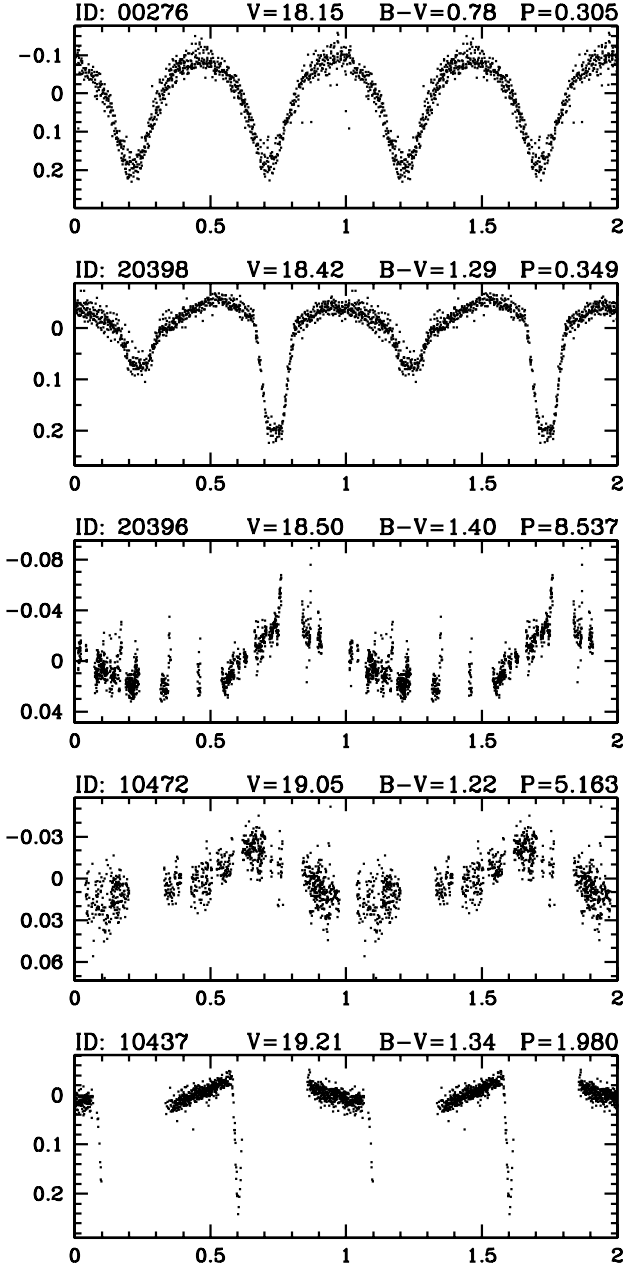

Phase

FIG. 4.-Same as Fig. 3, but for different objects.

with gray bars, and the light curve for the nearest object is shown in panel $c$, phased to the period of the variable. We show the phased light curve for the nearest source because some of the millimagnitude amplitude variables are visually blended. Having an object nearby with similar blending complications that does not reveal variability confirms the intrinsic nature of the low-amplitude variable. For completeness, we also show light curves in Figure 8 for two objects that do not survive the second-tier cuts but visually demonstrate intrinsic variability.

It should be noted that the DoPhot photometry detection software cannot successfully identify every object in the images. Objects that are too heavily blended, elongated, faint, saturated, etc., do not make it through the photometry pipeline. Therefore, the nearest object identified by the software may not be the nearest object to the variable. In most cases, however, the object identified as nearest is still close enough to the variable for the light-curve comparison to be useful.

We plot the properties of the potential cluster variables in Table 1 and the field variables in Table 2, with the two visually selected stars listed separately. For a variable to belong to the cluster it must have $B V I$ apparent magnitudes consistent with the cluster main sequence. For potential cluster members we give estimates for the stellar properties based on the CMD (see $\S 3$ ), and we classify their variable types (see $\S 5$ ) if possible. Both tables give magnitudes and positional data for all the variable stars.

\subsection{Matching to $2 M A S S$}

Most of the selected variables have matching counterparts in the Two Micron All Sky Survey (2MASS) database (Skrutskie et al. 1997), which we accessed through the VizieR Web portal. ${ }^{1}$ For variables with a 2 MASS counterpart within $1^{\prime \prime}$ radius, we list in Table 3 the 2MASS ID numbers, the 2MASS magnitudes in the $J, H$, and $K$ bands, and the distance between the variable positions we measure and the reported positions of the 2MASS sources. Three of the faintest stars, two cluster members and one field star, were not matched to any 2MASS object. A search of the literature revealed no known variable counterpart to any of the variable stars identified in this analysis. We were unable to find any published cases of variable stars in the observed field within the magnitude ranges we searched. We therefore conclude that all of the variables identified in this paper are previously unknown.

\section{VARIABLE CLASSIFICATION}

None of the variables we have discovered and listed in Tables 1 and 2 have been previously identified, according to searches in the SIMBAD and VizieR online catalogs and a search of the relevant literature. The large field of view, faint magnitude limit,

\footnotetext{
${ }^{1}$ The VizieR Web site is http://vizier.u-strasbg.fr/viz-bin/VizieR.
} 

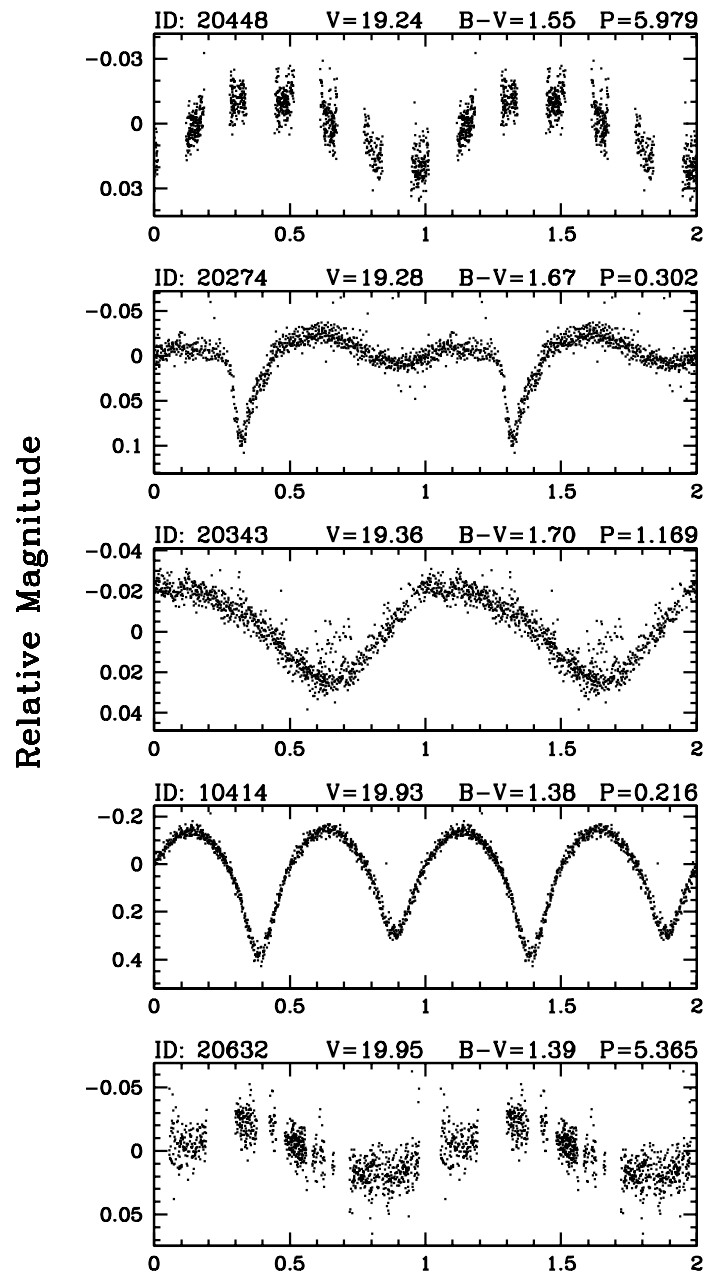
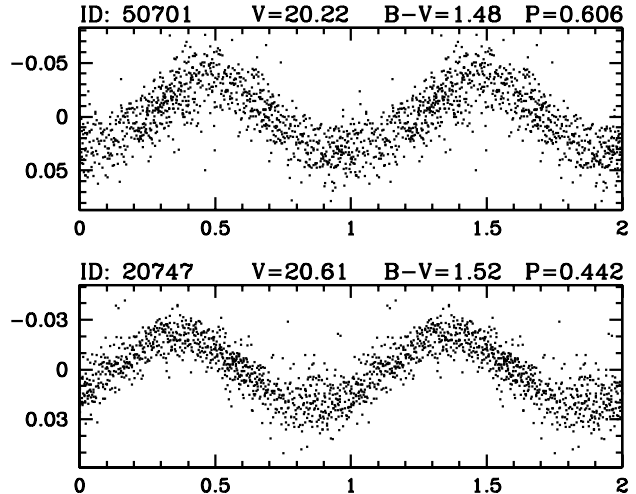

\section{Phase}

Fig. 5.-Same as Fig. 3, but for different objects.

frequent time sampling, and long observing baseline of STEPSS provide a thorough and unequaled exploration of the variable-star content for this cluster. STEPSS obtains $1 \%$ and $10 \%$ photometric precision from the saturation limit at $I \sim 15$ down to $I \sim 18$ and $\sim 20$, respectively. There are no other searches in NGC 1245 with the time baselines and magnitude limits appropriate for discovering the cluster variables. For that reason, there are no previously known variables that we would have expected to find that were not found.

Here, we identify those variables for which we believe we have sufficient information to classify as known variable types. We use the variable classification methods described by Sterken \& Jaschek (1996).

\subsection{Binaries}

Of the cluster members, objects 20176, 60193, 20534, 60303, and 10462 all have light curves characteristic of binary stars. Objects 60303 and 10462 are detached eclipsing binaries, designated as EA variables, or Algol-type binaries. Objects 20176, 60193, and 20534 are contact binaries, designated as EW variables, or W UMa-type binaries. Of the field variables, objects 60017, 20065, $30143,00276,20398,10437,20274$, and 10414 are all binary variables.

The periods and colors of contact binaries can be used as a rough distance indicator. A period-luminosity-color relation for contact binaries was developed by Rucinski (1994) and applied to contact binaries discovered in the core of the cluster 47 Tucanae by Albrow et al. (2001).

The relationship developed by Rucinski is

$$
M_{V}=-4.43 \log P+3.63(V-I)_{0}-0.31,
$$

where $P$ is in days. Using the derived $(V-I)_{0}$ colors (see $\S 3$ ) and the periods determined through the ANOVA algorithm, we calculate the distance modulus to the contact binary systems. Given that the distance modulus for the cluster is $12.27 \pm 0.12$, the Rucinski formula gives us a distance modulus of $(m-M)_{0}=$ 12.14 for object $20176,(m-M)_{0}=12.62$ for object 60193 , and $(m-M)_{0}=12.48$ for object 20534 , consistent with the classification of these objects as cluster members. The Rucinski relation has an intrinsic magnitude error of $\sim 0.3$, which is comparable to the systematic error found in Albrow et al. (2001).

\section{2. $\gamma$ Doradus Candidates}

Early F-type stars containing multiple frequencies with periods of $0.4-3$ days and $0.01-0.1 \mathrm{mag} V$-band amplitudes typify the $\gamma$ Doradus class (Krisciunas 1998; Kaye et al. 1999). The pulsation mechanism is thought to arise from high-order, lowdegree, nonradial gravity modes. The light curves for stars 70025, 


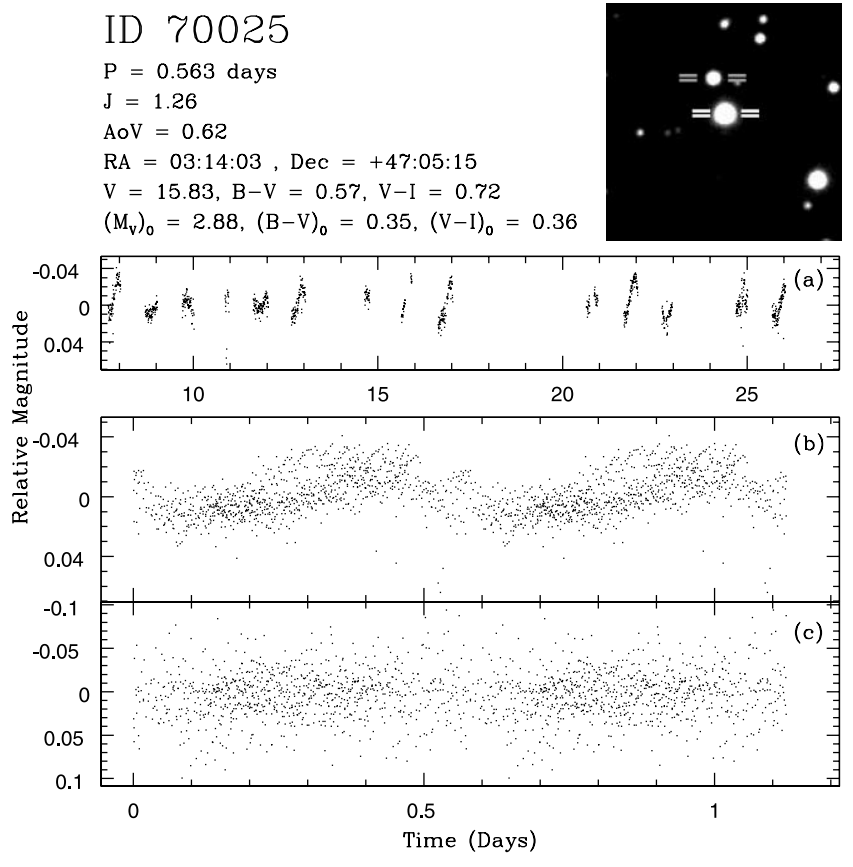

Fig. 6. - Light curves and finder charts for cluster variables. The first is for object 70025 . In the top right is the finder chart, with the variable marked with the white bars and the nearest detected object marked with gray bars. (a) Full unphased light curve for the variable. (b) Variable's light curve phased to the nearest period, with two periodic cycles plotted. (c) Light curve of the nearest object, phased to the period of the variable. [See the electronic edition of the Journal for Figs. 6.2-6.14.]

\section{ID 20053}

$P=3.173$ days

$\mathrm{J}=7.22$

$\mathrm{AoV}=0.95$

$\mathrm{RA}=03: 15: 11, \mathrm{Dec}=+47: 15: 02$

$\mathrm{V}=15.75, \mathrm{~B}-\mathrm{V}=0.94, \mathrm{~V}-\mathrm{I}=1.19$
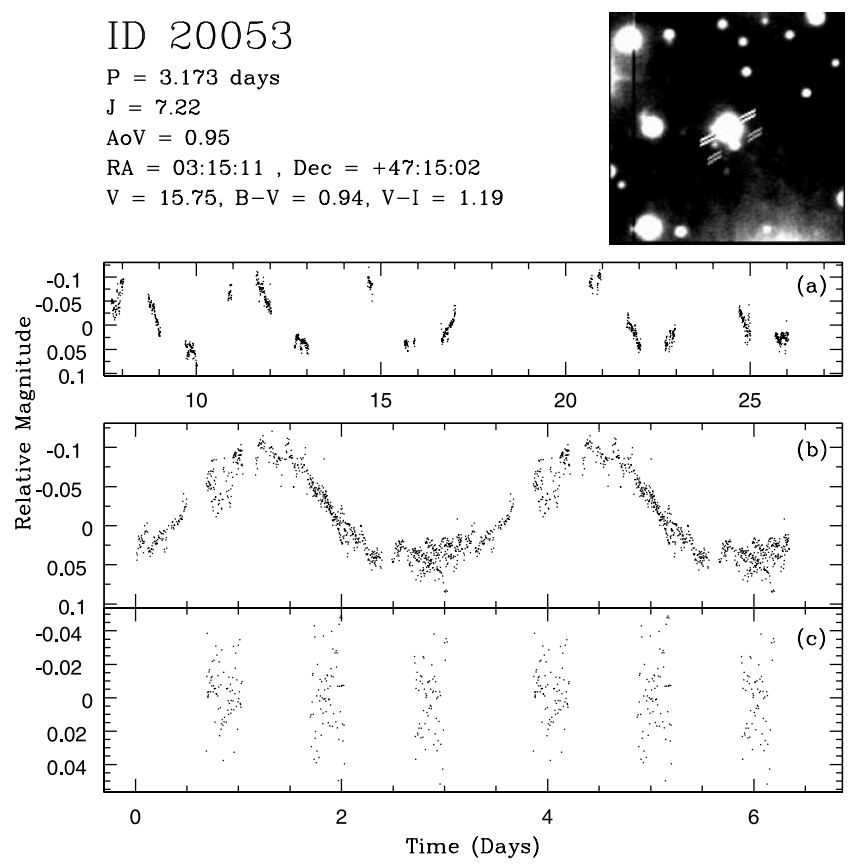

FIG. 7.- Light curves and finder charts for field variables. The first is for object 20053. In the top right is the finder chart, with the variable marked with the white bars and the nearest detected object marked with gray bars. (a) Full unphased light curve for the variable. (b) Variable's light curve phased to the nearest period, with two periodic cycles plotted. (c) Light curve of the nearest object, phased to the period of the variable. [See the electronic edition of the Journal for Figs. 7.2-7.27.]
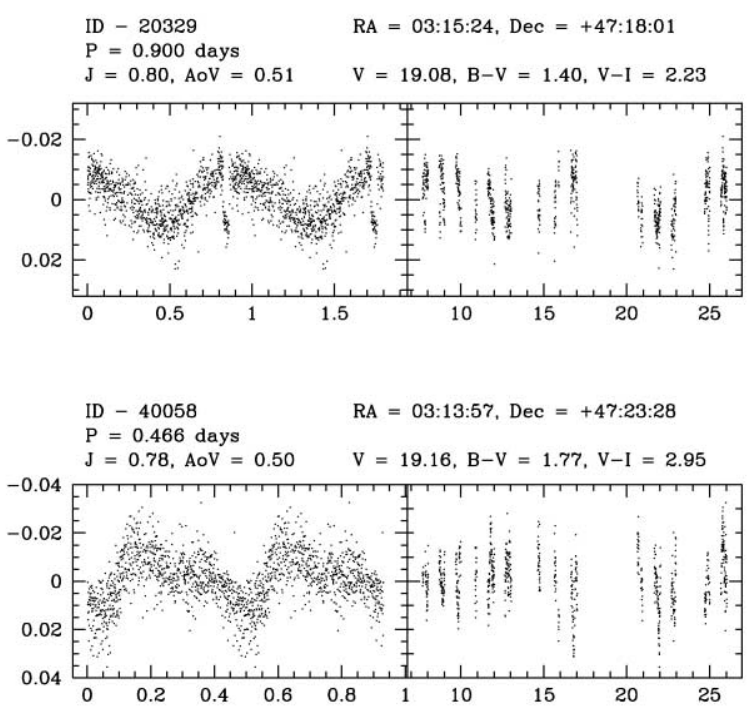

FIG. 8.- Light curves for two objects that did not survive the variability cuts but show coherent periodic variability on visual inspection. Phased light curves showing two full periods are shown on the left, and the full unphased light curves are shown on the right.

20196, 20223, and 20214 show evidence of $\gamma$ Doradus variability. We identify multiple frequencies using the successive leastsquares spectral analysis technique of Vaníček $(1969,1971)$ that is commonly applied to analyzing pulsating variables (Henry et al. 2005). Applying the spectral analysis to all the cluster variables, these four objects have unique signatures that set them apart from the others. For a majority of cluster variables, only the fundamental and harmonics exist in the light curve, whereas for the four $\gamma$ Doradus candidates, multiple frequencies exist that are not integer fractions of the fundamental.

Figures 9 and 10 present the light curves for the $\gamma$ Doradus candidates along with the best-fit light-curve models in the upper parts of the figures. Each subpanel shows a single night's data, and the lines represent models of the variability with a successively increased number of frequencies as identified in the spectral analysis. The solid line shows the first detected frequency fit to the data, the long-dashed line gives a two-component fit, and the short-dashed line gives a three-component fit. Figures 9 and 10 show the Vaníček (1971) optimum spectra statistic of the light curve as a function of frequency in the lower parts of the figures. The subpanels show successive identification of the frequencies present in the light curve from top to bottom. If a confident frequency is present, the frequency and its amplitude are noted in the upper right corner of the subpanel. We confidently detect a frequency when it appears in all variants of the light curves, which we explain next.

When applying the spectral analysis to the cluster binary variables 60193 and 20534, their high amplitude, short period, and regularity allow us to detect numerous frequencies that are all harmonics of the fundamental. However, in the original light curves lower significance frequencies were found very close to the dominant frequency detected. For binary stars this frequency splitting can occur if spot patterns on the close binaries appear or vary. The frequency splitting can also occur if the exposure timing is not accurate, or, as we discovered during this study, it can result from corrections to the light curves to reduce systematic errors. 
TABLE 1

Properties of Variable Potential Cluster Members

\begin{tabular}{|c|c|c|c|c|c|c|c|c|c|c|c|c|}
\hline STEPSS ID & $\left(M_{V}\right)_{0}{ }^{\mathrm{a}}$ & $(B-V)_{0}^{\mathrm{b}}$ & $(V-I)_{0}{ }^{\mathrm{b}}$ & R.A. (J2000.0) & Decl. (J2000.0) & Variable Type & $\begin{array}{c}M \\
\left(M_{\odot}\right)\end{array}$ & $\begin{array}{c}R \\
\left(R_{\odot}\right)\end{array}$ & $\begin{array}{c}\left(T_{\text {eff }}\right) \\
(\mathrm{K})\end{array}$ & $J$ & $\mathrm{AoV}$ & $\begin{array}{l}\text { Period } \\
\text { (days) }\end{array}$ \\
\hline $70025 \ldots \ldots \ldots \ldots$ & 2.88 & 0.35 & 0.36 & 031403 & +470515 & $\gamma$ Dor & 1.47 & 1.56 & 7000 & 1.262 & 0.619 & 0.563 \\
\hline $20196 \ldots \ldots \ldots \ldots$ & 3.18 & 0.36 & 0.42 & 031515 & +471419 & $\gamma$ Dor & 1.40 & 1.45 & 6800 & 0.774 & 0.566 & 0.408 \\
\hline $20223 \ldots \ldots \ldots \ldots$ & 3.32 & 0.40 & 0.42 & 031457 & +471543 & $\gamma$ Dor & 1.36 & 1.40 & 6700 & 1.470 & 0.619 & 1.068 \\
\hline $20214 \ldots \ldots \ldots \ldots$ & 3.32 & 0.44 & 0.48 & 031503 & +471826 & $\gamma$ Dor & 1.36 & 1.40 & 6700 & 0.877 & 0.604 & 1.163 \\
\hline $20176 \ldots \ldots \ldots \ldots$ & 3.67 & 0.44 & 0.51 & 031530 & +471437 & EW & 1.28 & 1.28 & 6500 & 1.352 & 0.656 & 0.301 \\
\hline 60193 ............... & 4.10 & 0.46 & 0.64 & 031428 & +471107 & EW & 1.19 & 1.16 & 6300 & 23.966 & 0.962 & 0.390 \\
\hline $00210 \ldots \ldots \ldots \ldots$ & 4.70 & 0.62 & 0.62 & 031448 & +470654 & $\ldots$ & 1.07 & 0.98 & 5900 & 9.515 & 0.977 & 0.201 \\
\hline $20534 \ldots \ldots \ldots \ldots$ & 5.36 & 0.82 & 0.85 & 031453 & +471632 & $\mathrm{EW}$ & 0.96 & 0.85 & 5600 & 4.777 & 0.960 & 0.281 \\
\hline $10429 \ldots \ldots \ldots \ldots$ & 5.57 & 0.73 & 0.75 & 031513 & +471116 & $\ldots$ & 0.93 & 0.82 & 5400 & 0.921 & 0.623 & 4.173 \\
\hline $20510 \ldots \ldots \ldots \ldots$ & 5.57 & 0.74 & 0.78 & 031506 & +471703 & $\ldots$ & 0.93 & 0.82 & 5400 & 0.827 & 0.584 & 10.32 \\
\hline $20513 \ldots \ldots \ldots \ldots$ & 5.69 & 0.89 & 0.92 & 031505 & +471509 & $\ldots$ & 0.91 & 0.80 & 5400 & 0.794 & 0.611 & 4.483 \\
\hline $60303 \ldots \ldots \ldots \ldots$ & 5.76 & 0.92 & 0.91 & 031435 & +470909 & EA & 0.89 & 0.79 & 5300 & 1.478 & 0.609 & 1.339 \\
\hline 30338............... & 6.11 & 0.84 & 0.87 & 031536 & +472304 & $\ldots$ & 0.85 & 0.75 & 5100 & 2.034 & 0.873 & 0.946 \\
\hline $10462 \ldots \ldots \ldots \ldots$ & 6.28 & 0.86 & 0.94 & 031456 & +471159 & EA & 0.83 & 0.73 & 5100 & 2.434 & 0.799 & 0.455 \\
\hline
\end{tabular}

Note.-Units of right ascension are hours, minutes, and seconds, and units of declination are degrees, arcminutes, and arcseconds.

${ }^{a}\left(M_{V}\right)_{0}$ was determined from observed $V$ magnitude using $A_{V}=0.68 \pm 0.09$ and a distance modulus of $12.27 \pm 0.12$ from Burke et al. (2004). Combined instrumental and systematic errors in $\left(M_{V}\right)_{0}$ are about $0.15 \mathrm{mag}$.

${ }^{\mathrm{b}}$ Dereddened values for $(B-V)_{0}$ and $(V-I)_{0}$ were determined using $A_{V}=0.68$ and $A_{I}=0.33$, with $R_{V}=3.2$, from Burke et al. (2004). Combined instrumental and systematic errors in the colors are about 0.1 mag in $(B-V)_{0}$ and 0.15 in $(V-I)_{0}$.

TABLE 2

Properties of Variable Field Stars

\begin{tabular}{|c|c|c|c|c|c|c|c|c|}
\hline STEPSS ID & $V^{\mathrm{a}}$ & $B-V^{\mathrm{a}}$ & $V-I^{\mathrm{b}}$ & R.A. (J2000.0) & Decl. (J2000.0) & $J$ & AoV & $\begin{array}{l}\text { Period } \\
\text { (days) }\end{array}$ \\
\hline $20053 \ldots \ldots \ldots$ & 15.75 & 0.95 & 1.18 & 031511 & +471502 & 7.223 & 0.945 & 3.173 \\
\hline $60017 \ldots \ldots \ldots$ & 15.98 & 0.82 & 0.95 & 031351 & +470821 & 6.974 & 0.924 & 0.361 \\
\hline 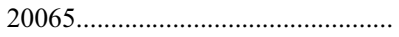 & 16.28 & 0.99 & 1.20 & 031504 & +471433 & 7.879 & 0.707 & 6.016 \\
\hline 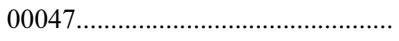 & 16.59 & 0.91 & 1.06 & 031520 & +470601 & 2.770 & 0.931 & 2.869 \\
\hline $60076 \ldots \ldots . .$. & 16.62 & 0.83 & 1.03 & 031352 & +470850 & 1.118 & 0.736 & 8.618 \\
\hline 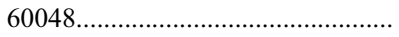 & 16.80 & 1.20 & 1.48 & 031429 & +471041 & 1.979 & 0.721 & 14.40 \\
\hline 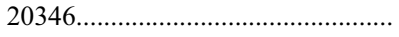 & 16.91 & 0.49 & 0.71 & 031520 & +471924 & 2.387 & 0.876 & 2.879 \\
\hline $50028 \ldots \ldots$. & 17.04 & 1.21 & 1.39 & 031349 & +471603 & 0.808 & 0.652 & 6.361 \\
\hline 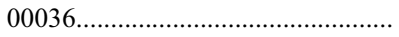 & 17.24 & 1.33 & 1.52 & 031528 & +470427 & 0.856 & 0.784 & 11.27 \\
\hline 50209 & 17.33 & 0.61 & 0.84 & 031351 & +471426 & 1.901 & 0.916 & 0.936 \\
\hline $20237 \ldots \ldots$ & 17.59 & 1.50 & 1.95 & 031452 & +471925 & 1.887 & 0.846 & 7.306 \\
\hline 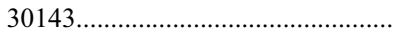 & 17.69 & 1.30 & 1.29 & 031541 & +472601 & 6.753 & 0.974 & 0.252 \\
\hline 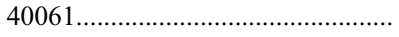 & 17.72 & 1.51 & 2.06 & 031407 & +472203 & 2.191 & 0.868 & 1.130 \\
\hline 70108 & 17.99 & 1.07 & 1.35 & 031404 & +470322 & 1.019 & 0.711 & 1.822 \\
\hline 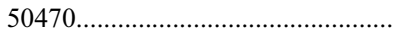 & 18.10 & 0.62 & 0.86 & 031428 & +471636 & 0.843 & 0.666 & 12.03 \\
\hline 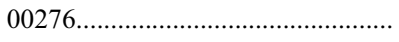 & 18.15 & 0.78 & 1.06 & 031537 & +470445 & 8.737 & 0.953 & 0.304 \\
\hline $20398 \ldots \ldots . .$. & 18.42 & 1.29 & 2.00 & 031450 & +471603 & 7.660 & 0.885 & 0.349 \\
\hline 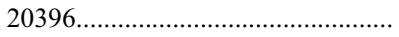 & 18.50 & 1.40 & 1.73 & 031450 & +471525 & 2.449 & 0.731 & 8.537 \\
\hline 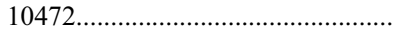 & 19.05 & 1.23 & 1.45 & 031454 & +471252 & 0.939 & 0.688 & 5.163 \\
\hline 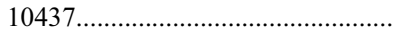 & 19.21 & 1.34 & 1.72 & 031508 & +470949 & 1.145 & 0.633 & 1.980 \\
\hline 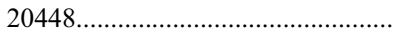 & 19.24 & 1.56 & 2.02 & 031536 & +471709 & 1.349 & 0.764 & 5.979 \\
\hline 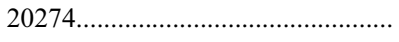 & 19.28 & 1.66 & 3.37 & 031436 & +471929 & 2.880 & 0.854 & 0.302 \\
\hline 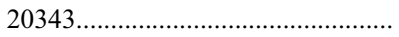 & 19.36 & 1.70 & 3.09 & 031523 & +471843 & 2.758 & 0.899 & 1.169 \\
\hline 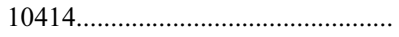 & 19.93 & 1.38 & 1.82 & 031519 & +470932 & 10.143 & 0.980 & 0.216 \\
\hline 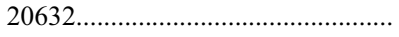 & 19.95 & 1.39 & 1.78 & 031524 & +471956 & 1.051 & 0.700 & 5.365 \\
\hline 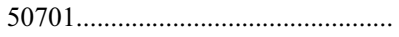 & 20.22 & 1.48 & 1.95 & 031404 & +471620 & 1.111 & 0.682 & 0.606 \\
\hline 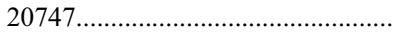 & 20.61 & 1.52 & 3.11 & 031515 & +471529 & 1.056 & 0.756 & 0.442 \\
\hline 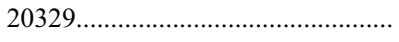 & 19.08 & 1.40 & 2.23 & 031524 & +471801 & 0.796 & 0.511 & 0.900 \\
\hline 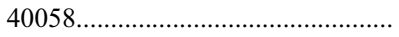 & 19.16 & 1.77 & 2.95 & 031357 & +472328 & 0.782 & 0.503 & 0.931 \\
\hline
\end{tabular}

Note.-Units of right ascension are hours, minutes, and seconds, and units of declination are degrees, arcminutes, and arcseconds.

a Combined instrumental and systematic errors in absolute $B$ and $V$ magnitudes are 0.05 mag.

${ }^{\mathrm{b}}$ Combined instrumental and systematic errors in absolute $I$ magnitude are $0.1 \mathrm{mag}$. 
TABLE 3

Variable Matches to 2MASS

\begin{tabular}{|c|c|c|c|c|c|}
\hline STEPSS ID & 2MASS ID & $\begin{array}{l}\text { 2MASS Offset } \\
\quad(\operatorname{arcsec})\end{array}$ & $J$ & $H$ & $K$ \\
\hline \multicolumn{6}{|c|}{ Cluster Members } \\
\hline $00210 \ldots \ldots \ldots \ldots \ldots \ldots$ & $03144786+4706544$ & 0.098 & $16.116 \pm 0.094$ & $15.670 \pm 0.120$ & $15.881 \pm 0.065$ \\
\hline 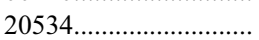 & $03145325+4716321$ & 0.579 & $16.270 \pm 0.107$ & $15.683 \pm 0.124$ & $15.512 \pm 0.172$ \\
\hline $20176 \ldots \ldots \ldots \ldots \ldots \ldots \ldots$ & $03152956+4714374$ & 0.066 & $15.240 \pm 0.044$ & $14.901 \pm 0.059$ & $14.677 \pm 0.069$ \\
\hline 60193 & $03142811+4711067$ & 0.079 & $15.757 \pm 0.072$ & $15.245 \pm 0.091$ & $15.348 \pm 0.158$ \\
\hline 20196 & $03151490+4714185$ & 0.043 & $14.904 \pm 0.030$ & $14.670 \pm 0.051$ & $14.555 \pm 0.060$ \\
\hline $10429 \ldots \ldots \ldots \ldots \ldots \ldots$ & $03151250+4711157$ & 0.232 & $16.635 \pm 0.149$ & $15.760 \pm 0.068$ & $14.873 \pm 0.101$ \\
\hline $10462 \ldots \ldots \ldots \ldots \ldots$ & & $\ldots$ & & & \\
\hline 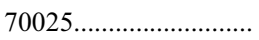 & $03140339+4705144$ & 0.115 & $14.747 \pm 0.040$ & $14.509 \pm 0.051$ & $14.483 \pm 0.069$ \\
\hline 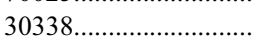 & $\ldots$ & $\ldots$ & $\ldots$ & & $\ldots$ \\
\hline 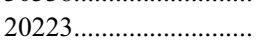 & $03145747+4715430$ & 0.063 & $14.922 \pm 0.043$ & $14.733 \pm 0.065$ & $14.531 \pm 0.080$ \\
\hline $20214 \ldots \ldots \ldots \ldots \ldots \ldots \ldots$ & $03150277+4718258$ & 0.063 & $14.939 \pm 0.033$ & $14.621 \pm 0.048$ & $14.598 \pm 0.077$ \\
\hline $60303 \ldots \ldots \ldots \ldots \ldots \ldots \ldots$ & $03143504+4709095$ & 0.363 & $16.608 \pm 0.150$ & $16.140 \pm 0.180$ & $15.785 \pm 0.216$ \\
\hline 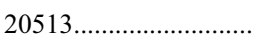 & $03150464+4715090$ & 0.356 & $16.438 \pm 0.117$ & $15.938 \pm 0.153$ & $16.060 \pm 0.030$ \\
\hline $20510 \ldots \ldots \ldots \ldots \ldots \ldots$ & $03150636+4717030$ & 0.572 & $16.396 \pm 0.116$ & $16.033 \pm 0.168$ & $15.821 \pm 0.230$ \\
\hline \multicolumn{6}{|c|}{ Field Stars } \\
\hline $10414 \ldots \ldots \ldots \ldots \ldots \ldots \ldots . .$. & $03151946+4709324$ & 0.483 & $17.050 \pm 0.151$ & $16.311 \pm 0.173$ & $16.243 \pm 0.283$ \\
\hline 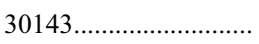 & $03154104+4726009$ & 0.109 & $15.495 \pm 0.048$ & $14.874 \pm 0.064$ & $14.804 \pm 0.095$ \\
\hline 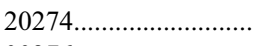 & $03143588+4719290$ & 0.056 & $14.097 \pm 0.025$ & $13.486 \pm 0.027$ & $13.140 \pm 0.030$ \\
\hline $00276 \ldots \ldots \ldots$ & $03153653+4704457$ & 0.483 & $16.386 \pm 0.096$ & $15.946 \pm 0.156$ & $15.708 \pm 0.198$ \\
\hline 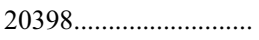 & $03144954+4716030$ & 0.124 & $15.156 \pm 0.049$ & $14.508 \pm 0.053$ & $14.259 \pm 0.061$ \\
\hline $60017 \ldots \ldots \ldots \ldots \ldots$ & $03135090+4708209$ & 0.079 & $14.449 \pm 0.034$ & $14.054 \pm 0.045$ & $13.983 \pm 0.049$ \\
\hline $20747 \ldots \ldots \ldots \ldots \ldots \ldots \ldots$ & $03151453+4715287$ & 0.051 & $15.779 \pm 0.074$ & $15.178 \pm 0.079$ & $14.925 \pm 0.088$ \\
\hline $50701 \ldots \ldots$ & $\ldots$ & $\ldots$ & $\ldots$ & $\ldots$ & $\ldots$ \\
\hline $50209 \ldots \ldots \ldots \ldots \ldots \ldots \ldots$ & $03135130+4714258$ & 0.123 & $15.984 \pm 0.078$ & $15.727 \pm 0.130$ & $15.145 \pm 0.135$ \\
\hline 40061........................ & $03140666+4722034$ & 0.094 & $14.462 \pm 0.031$ & $13.657 \pm 0.033$ & $13.494 \pm 0.040$ \\
\hline $20343 \ldots \ldots \ldots \ldots \ldots \ldots \ldots . .$. & $03152256+4718432$ & 0.027 & $14.618 \pm 0.029$ & $14.022 \pm 0.042$ & $13.669 \pm 0.033$ \\
\hline 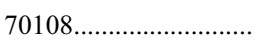 & $03140379+4703222$ & 0.131 & $15.811 \pm 0.080$ & $15.183 \pm 0.085$ & $15.317 \pm 0.141$ \\
\hline 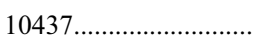 & $03150798+4709492$ & 0.192 & $16.476 \pm 0.136$ & $15.894 \pm 0.151$ & $15.556 \pm 0.188$ \\
\hline 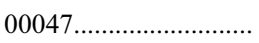 & $03152045+4706012$ & 0.017 & $14.861 \pm 0.036$ & $14.504 \pm 0.046$ & $14.269 \pm 0.051$ \\
\hline 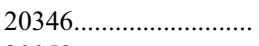 & $03152015+4719237$ & 0.263 & $15.522 \pm 0.054$ & $15.184 \pm 0.095$ & $15.166 \pm 0.112$ \\
\hline $20053 \ldots \ldots \ldots$ & $03151087+4715025$ & 0.109 & $13.631 \pm 0.021$ & $13.062 \pm 0.024$ & $12.925 \pm 0.030$ \\
\hline 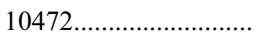 & $03145353+4712519$ & 0.375 & $16.463 \pm 0.125$ & $16.133 \pm 0.171$ & $15.698 \pm 0.190$ \\
\hline $20632 \ldots \ldots \ldots \ldots \ldots \ldots \ldots$ & $03152440+4719552$ & 0.756 & $16.839 \pm 0.175$ & $15.903 \pm 0.037$ & $13.837 \pm 0.039$ \\
\hline 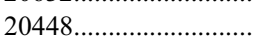 & $03153626+4717089$ & 0.044 & $16.061 \pm 0.074$ & $15.448 \pm 0.102$ & $15.166 \pm 0.103$ \\
\hline 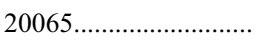 & $03150383+4714329$ & 0.033 & $13.915 \pm 0.022$ & $13.377 \pm 0.025$ & $13.261 \pm 0.029$ \\
\hline 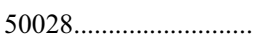 & $03134853+4716027$ & 0.033 & $14.791 \pm 0.036$ & $14.170 \pm 0.040$ & $14.004 \pm 0.050$ \\
\hline 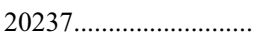 & $03145177+4719247$ & 0.044 & $14.407 \pm 0.036$ & $13.652 \pm 0.032$ & $13.471 \pm 0.035$ \\
\hline 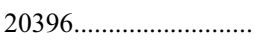 & $03145015+4715251$ & 0.089 & $15.666 \pm 0.067$ & $14.892 \pm 0.056$ & $14.631 \pm 0.071$ \\
\hline 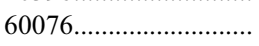 & $03135202+4708503$ & 0.099 & $14.875 \pm 0.036$ & $14.507 \pm 0.053$ & $14.238 \pm 0.060$ \\
\hline 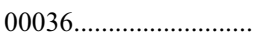 & $03152825+4704264$ & 0.070 & $14.739 \pm 0.056$ & $14.132 \pm 0.051$ & $13.948 \pm 0.051$ \\
\hline 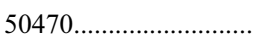 & $03142814+4716366$ & 0.300 & $16.370 \pm 0.121$ & $15.750 \pm 0.091$ & $15.348 \pm 0.158$ \\
\hline 60048........................... & $03142872+4710408$ & 0.029 & $14.248 \pm 0.029$ & $13.589 \pm 0.028$ & $13.399 \pm 0.035$ \\
\hline $20329 \ldots \ldots \ldots \ldots \ldots \ldots \ldots$ & $03152445+4718006$ & 0.077 & $15.542 \pm 0.054$ & $14.841 \pm 0.064$ & $14.528 \pm 0.063$ \\
\hline 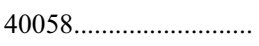 & $03135669+4723282$ & 0.135 & $14.692 \pm 0.037$ & $14.046 \pm 0.037$ & $13.690 \pm 0.041$ \\
\hline
\end{tabular}

We find that the procedure for removing the systematic trend in a light curve performs less than ideally in the presence of high amplitude variability. It slightly distorts the light curve enough to give rise to frequency splitting. The close frequency splitting is reduced when analyzing light curves without the seeing trend correction. In addition, the frequency splitting is further reduced by eliminating the last two nights of data. This suggests that the timing may be an issue over the baseline of observations. However, considering that the last two nights had some of the best seeing, it is not clear which of these two factors is the cause of the frequency splitting. Overall, the cleanest spectral analysis-which detects only harmonics - for stars 60193 and 20534 occurs without the seeing correction and without the last two nights of data. Thus, we define confidently detected frequencies in our $\gamma$ Doradus candidates as ones that are stable and detected in all variants of the light curves: the original light curves, light curves without the seeing correction, light curves without the last two nights of data, and light curves without the seeing correction and without the last two nights of data.

In addition to these four objects having confidently identified multiple frequencies that are not harmonically related, they also occupy the upper main sequence in the CMD, as expected for the typical spectral type for $\gamma$ Doradus variables. The large circles in Figure 1 designate their locations in the CMD. However, the $B-V$ and estimated temperature for candidates 20214 and 20223 ( $B-V \sim 0.42$ and $T_{\text {eff }} \sim 6700 \mathrm{~K}$ ) are redward and cooler than the empirically determined cool-edge boundary for $\gamma$ Doradus variables as defined by Henry et al. (2005) $(B-V=0.38)$. The 

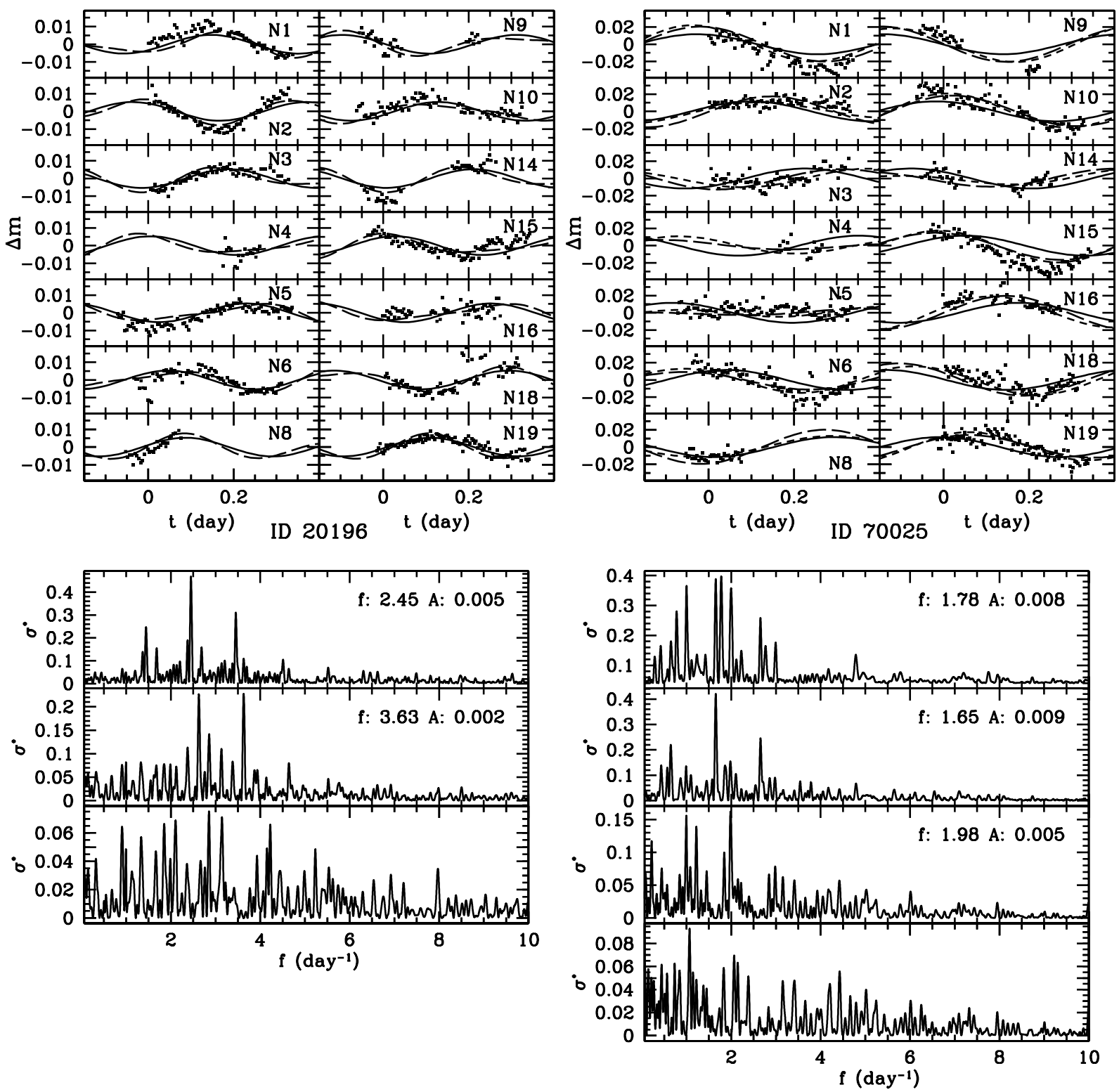

FIG. 9.- Light curves and power spectra for $\gamma$ Doradus candidates 20196 and 70025. In the light-curve plots in the top left and top right, each subpanel shows a single night's observations, with a label in the upper right corner of each subpanel identifying the night of the observing run and an arbitrary $x$-axis zero point. The solid line shows a single-component fit to the light curve, the long-dashed line gives a two-component fit, and the short-dashed line gives a three-component fit. The bottom left and bottom right panels show successive optimum spectra for 20196 and 70025 . The upper subpanel shows the spectrum of the light curve that identifies the strongest frequency component present in the data. The component's frequency and amplitude are labeled in the upper right corner of the subpanel. The successive subpanels from top to bottom show the spectra after taking into account the known constituents identified in the previous panels. The bottom subpanel gives the spectra after taking into account the two previously identified components. No stable component is identified in the bottom subpanel.

empirical cool edge of Henry et al. (2005) agrees well with the theoretical $\gamma$ Doradus instability strip as given by Warner et al. (2003) $\left(T_{\text {eff }}=6850 \mathrm{~K}\right)$.

Alternatively, our effective temperatures may be systematically incorrect. The effective temperatures for these objects are estimated from the CMD and the best-fit cluster isochrone as described in Burke et al. (2004). Varying the metallicity of the cluster for $-0.26 \leq[\mathrm{Fe} / \mathrm{H}] \leq+0.13$ while minimizing age, distance, and reddening to yield a best-fit isochrone to the observed CMD yields a maximum $T_{\text {eff }}=6800 \mathrm{~K}$ for these two stars. Reaching this high temperature requires an implausible $[\mathrm{Fe} / \mathrm{H}]=+0.13$. Thus, if objects 20214 and 20223 are confirmed as $\gamma$ Doradus stars they may represent the coolest known members of this class of variables. Also, the $\gamma$ Doradus candidates discovered in this study may represent the oldest known members of this class of variables, given the $\sim 1$ Gyr age for NGC 1245 (Martín \& Rodríguez 2002).

Confirming the $\gamma$ Doradus variables will require follow-up spectroscopy and simultaneous light curves in several passbands. However, alternative models for the variability cannot reproduce the multiple frequencies that are not harmonically related (Krisciunas 1998). This is especially the case for objects 70025 and 20214, for which we confidently detect three components that are not harmonically related. In addition, estimating the physical parameters of the stars from the best-fit isochrone, we calculate the pulsation constant to range over $-0.57<\log Q<$ -0.39 for our candidates. These values are consistent with the confirmed $\gamma$ Doradus variables of Handler \& Shobbrook (2002). We used the first strong frequency detected when calculating the pulsation constant. 

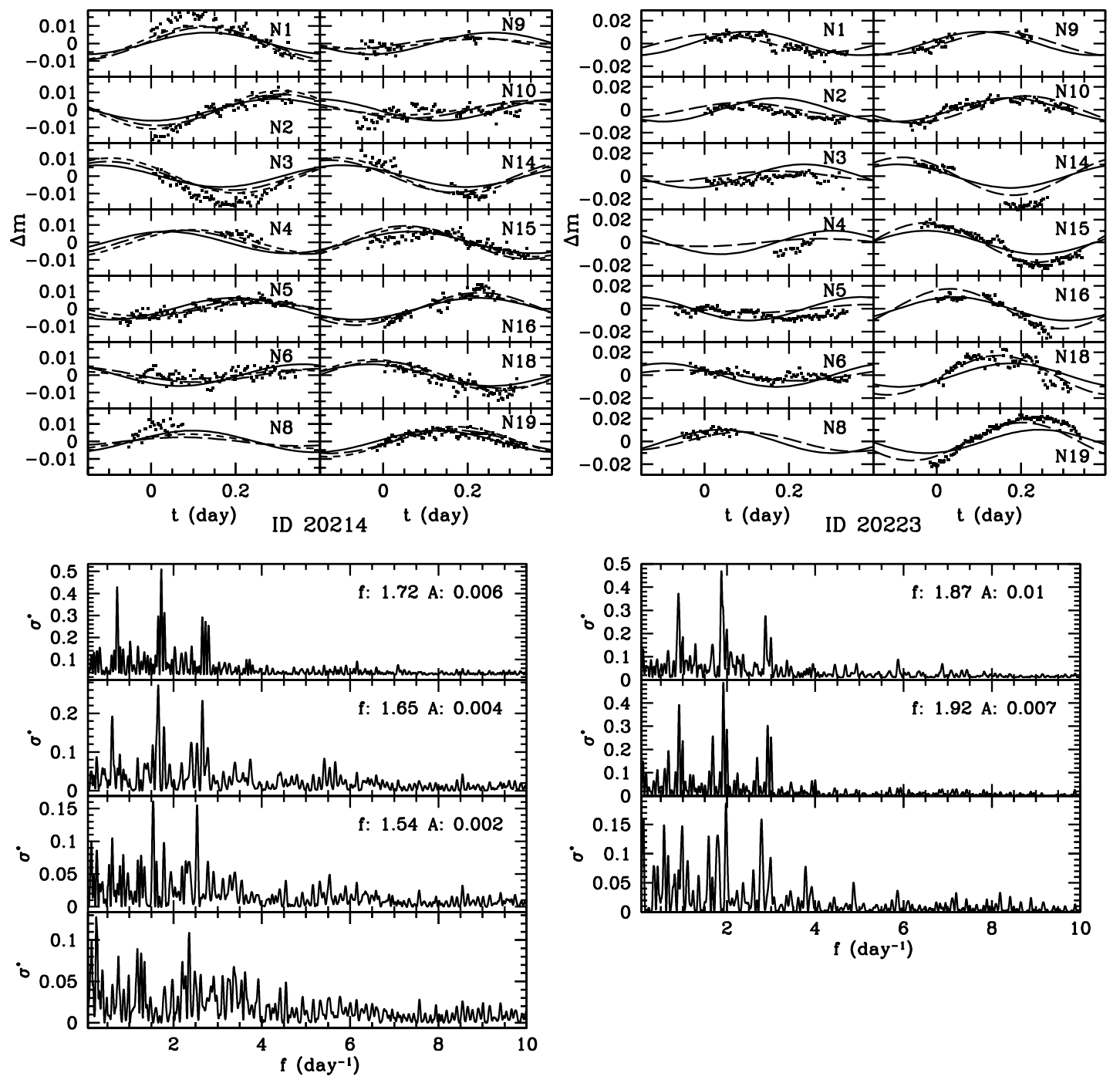

FIG. 10.-Same as Fig. 9, but for $\gamma$ Doradus candidates 20214 and 20223.

Assuming these four objects are $\gamma$ Doradus variables, we are able to measure the fraction of cluster members exhibiting this type of variability. Burke et al. (2004) statistically determine the cluster membership by characterizing the background star counts as a function of apparent magnitude from a control field at the outskirts of the field of view. The cluster membership as a function of magnitude is shown as Figure 9 in Burke et al. (2005). There are an estimated 46 cluster members for NGC 1245 for $15.83<V<$ 16.27, the apparent magnitudes of our brightest and faintest $\gamma$ Doradus candidates. This corresponds to $2.88<\left(M_{V}\right)_{0}<3.32$, assuming a cluster distance modulus $(m-M)_{0}=12.27$ and extinction $A_{V}=0.68$. If all four objects are confirmed as $\gamma$ Doradus variables, then $8.7 \%$ of stars with properties similar to NGC 1245 in the above absolute magnitude range exhibit $\gamma$ Doradus variability. If none of the objects end up being $\gamma$ Doradus variables, then a null result implies that $<6.5 \%$ ( $95 \%$ confidence) of stars in this magnitude range exhibit $\gamma$ Doradus variability, assuming we are $100 \%$ complete in detecting variability with amplitudes similar to the candidates.

\section{CONCLUSIONS}

We have discovered 43 previously unknown variables in the field of NGC 1245, of which 14 are potential cluster members. Many of these variables have very low amplitude. Our method for determining relative photometry allows us to achieve precision to less than $1 \%$ for stars brighter than $V=18$, enabling the characterization of such low-amplitude variables. The techniques described in this paper can be readily applied to other long-term photometric data sets to determine the variability content of other surveys.

We roughly characterize the variables potentially belonging to the cluster. The light curves for objects 20534, 20176, 60193, 10462, and 60303 contain strong evidence for binarity. Objects 10462 and 60303 are detached eclipsing binaries. If their membership in this cluster is confirmed, then the known ages and composition of these binaries offer opportunities for testing stellar models for cool stars.

The light curves for objects 20196, 70025, 20223, and 20214 show evidence that multiple frequencies are present and located near the main-sequence turnoff. These objects are possible $\gamma$ Doradus candidates. Objects 20223 and 20214 will be the coolest $\left(T_{\text {eff }} \sim 6700 \mathrm{~K}\right)$ known $\gamma$ Doradus variables if their status is verified. The standard solar metallicity and solar-calibrated mixing length models of $\gamma$ Doradus variability do not predict pulsations in stars this cool (Dupret et al. 2004; Warner et al. 2003). Further exploration of parameter space may be necessary to fit these cool $\gamma$ Doradus candidates. Alternatively, the variability for these four objects may result from rapid rotation coupled with unstable spot patterns on their surfaces. Measuring the rotation rates and simultaneous light curves in multiple passbands for these objects would settle their classification. 
The natures of potential cluster members $00210,30338,10429$, 20513 , and 20510 remain uncertain. These objects most likely result from ellipsoidal variability. Of the noncluster objects, 10414 , 30143, 20274, 20398, 10437, and 20065 are eclipsing binaries. The rest of the nonmembers cannot be well characterized without knowledge of their absolute brightnesses and colors.
We would like to thank Marc Pinsonneault, Darren DePoy, Kris Stanek, and Rick Pogge for helpful comments. We thank Christopher Alard for the implementation of the ANOVA period analysis. This work was supported by the National Aeronautics and Space Administration under grant NNG 04GO70G issued through the Origins of Solar Systems program.
Albrow, M. D., Gilliland, R. L., Brown, T. M., Edmonds, P. D., Guhathakurta, P., \& Sarajedini, A. 2001, ApJ, 559, 1060

Bramich, D. M., et al. 2005, MNRAS, 359, 1096

Burke, C. J., DePoy, D. L., Gaudi, B. S., Marshall, J. L., \& Pogge, R. W. 2002, in ASP Conf. Ser. 294, Scientific Frontiers in Research on Extrasolar Planets, ed. D. Deming \& S. Seager (San Francisco: ASP), 379

Burke, C. J., Gaudi, B. S., DePoy, D. L., \& Pogge, R. W. 2005, AJ, 132, 210

Burke, C. J., Gaudi, B. S., DePoy, D. L., Pogge, R. W., \& Pinsonneault, M. 2004, AJ, 127, 2382

Dupret, M.-A., Grigahcène, A., Garrido, R., Gabriel, M., \& Scuflaire, R. 2004, A\&A, 414, L17

Eyer, L., \& Blake, C. 2005, MNRAS, 358, 30

Handler, G., \& Shobbrook, R. R. 2002, MNRAS, 333, 251

Hargis, J. R., Sandquist, E. L., \& Bradstreet, D. H. 2004, BAAS, 205, 22.12

Henry, G. W., Fekel, F. C., \& Henry, S. M. 2005, AJ, 129, 2815

Hidas, M. G., et al. 2005, MNRAS, 360, 703

Horne, K. 2003, in ASP Conf. Ser. 294, Scientific Frontiers in Research on Extrasolar Planets, ed. D. Deming \& S. Seager (San Francisco: ASP), 361

Kaluzny, J., Stanek, K. Z., Krockenberger, M., Sasselov, D. D., Tonry, J. L., \& Mateo, M. 1998, AJ, 115, 1016

Kaye, A. B., Handler, G., Krisciunas, K., Poretti, E., \& Zerbi, F. M. 1999, PASP, 111, 840

Krisciunas, K. 1998, in IAU Symp. 185, New Eyes to See Inside the Sun and Stars, ed. F.-L. Deubner, J. Christensen-Dalsgaard, \& D. Kurtz (Dordrecht: Kluwer), 339
Martín, S., \& Rodríguez, E. 2002, in ASP Conf. Ser. 259, Radial and Nonradial Pulsations as Probes of Stellar Physics, ed. C. Aerts, T. R. Bedding, \& J. Christensen-Dalsgaard (San Francisco: ASP), 152

Mizerski, T., \& Bejger, M. 2002, Acta Astron., 52, 61

Mochejska, B. J., Stanek, K. Z., Sasselov, D. D., \& Szentgyorgyi, A. H. 2002, AJ, 123, 3460

Mochejska, B. J., Stanek, K. Z., Sasselov, D. D., Szentgyorgyi, A. H., Westover, M., \& Winn, J. N. 2004, AJ, 128, 312

Mochejska, B. J., et al. 2005, AJ, 129, 2856

Rucinski, S. M. 1994, PASP, 106, 462

Schwarzenberg-Czerny, A. 1996, ApJ, 460, L107

Skrutskie, M. F., et al. 1997, in The Impact of Large-Scale Near-IR Sky Surveys, ed. F. Garzon et al. (Dordrecht: Kluwer), 25

Sterken, C., \& Jaschek, C. 1996, Light Curves of Variable Stars: A Pictoral Atlas (Cambridge: Cambridge Univ. Press)

Stetson, P. B. 1996, PASP, 108, 851

Street, R. A., et al. 2003, MNRAS, 340, 1287

Vaníček, P. 1969, Ap\&SS, 4, 387 1971, Ap\&SS, 12, 10

von Braun, K., Lee, B. L., Seager, S., Yee, H. K. C., Mallén-Ornelas, G., \& Gladders, M. D. 2005, PASP, 117, 141

Warner, P. B., Kaye, A. B., \& Guzik, J. A. 2003, ApJ, 593, 1049

Yi, S., Demarque, P., Kim, Y. C., Lee, Y. W., Ree, C. H., Lejeune, T., \& Barnes, S. 2001, ApJS, 136, 417 